\title{
Genealogia acadêmica e sua relação com a trajetória do conhecimento na Escola Nacional de Saúde Pública Sergio Arouca, Fundação Oswaldo Cruz
}

\author{
Academic genealogy and its relationship with the trajectory \\ of knowledge in the National School of Public Health \\ Sergio Arouca, Oswaldo Cruz Foundation
}

\author{
Laura Cristina Simões VIANA 1 (D) 0000-0003-1454-5231 \\ Sheila Maria Ferraz Mendonça de SOUZA' 1 iD 0000-0001-5054-0126 \\ Rafael Jeferson Pezzuto DAMACENO2 (iD 0000-0003-4910-1534 \\ Jesús Pascual MENA-CHALCO² ${ }^{\text {iD } 0000-0001-7509-5532}$
}

\begin{abstract}
Resumo
A genealogia acadêmica hierarquiza as relações formais de orientação em redes de pares orientador-orientado, por meio das quais é possível realizar uma série de análises, tais como identificar quem são os acadêmicos mais influentes, em quais áreas eles atuam, e qual é a relação existente entre essas áreas. Este trabalho tem como objetivo construir e analisar a genealogia acadêmica da Escola Nacional de Saúde Pública Sergio Arouca, da Fundação Oswaldo Cruz. Para tanto, foram utilizados os registros da rede de bibliotecas dessa instituição e os currículos da Plataforma Lattes dos pesquisadores doutores, orientadores e orientados, listados nesses registros. Os resultados obtidos indicam que, na instituição analisada, o conhecimento acadêmico tem origens múltiplas, as quais remontam à primeira metade do século vinte, e que a trajetória do conhecimento em Saúde Pública é multidisciplinar. Atualmente, o conhecimento acadêmico na Instituição tem a marca da urgência do ambiente, no sentido de obter respostas sobre/para a realidade da saúde no contexto brasileiro.
\end{abstract}

Palavras-chave: Genealogia acadêmica. Indicadores em ciência e tecnologia. Mapeamento da ciência. Saúde pública.

\begin{abstract}
Academic genealogy hierarchizes formal advisor-advisee relationships in networks of students and their advisors providing room for a series of analyzes, such as identifying who are the most influential academics, in what areas they act, and what is the relationship between these areas. This work aims to build and analyze the academic genealogy of the National School of Public Health Sergio Arouca, of the Oswaldo Cruz Foundation. Therefore, this work made use of the PhD mentoring records of the library of this institution

\footnotetext{
1 Fundação Oswaldo Cruz, Escola Nacional de Saúde Pública Sergio Arouca, Vice-Direção de Pesquisa e Inovação. R. Leopoldo Bulhões, 1480, Bonsucesso, 21031-210, Rio de Janeiro, RJ, Brasil. Correspondência para/Correspondence to: L.C.S. VIANA. E-mail: <laura@fiocruz.br>

2 Universidade Federal do ABC, Centro de Matemática, Computação e Cognição, Departamento de Ciência da Computação. Santo André, SP, Brasil.

Recebido em 24 de outubro de 2018 e aprovado em 15 de março de 2019.
}

Como citar este artigo/How to cite this article

Viana, L.C.S. et al. Genealogia acadêmica e sua relação com a trajetória do conhecimento na Escola Nacional de Saúde Pública Sergio Arouca, Fundação Oswaldo Cruz. Transinformação, 31, e180073, 2019. http://dx.doi.org/10.1590/1678-9865201931e180073 
and of the researchers' curriculum from Lattes Platform. The results showed that, in the analyzed institution, academic knowledge has multiple origins, which dated to the first half of the twentieth century, and that the trajectory of knowledge in Public Health is multidisciplinary. Currently, the academic knowledge in the institution has the mark of the urgency of the environment, meaning that it is built to obtain answers about and to the reality of health in the Brazilian context.

Keywords: Academic genealogy. Science and technology indicators. Science mapping. Public health.

\section{Introdução}

Com frequência, avalia-se o impacto científico de um acadêmico por meio de sua produção científica, contabilizando, por exemplo, o número de artigos publicados e de artigos citados nos últimos anos, bem como outras medidas bibliométricas. Por outro lado, recentemente, iniciativas têm incorporado nessa avaliação a formação de recursos humanos, ou seja, a quantidade e a qualidade da formação de novos cientistas oriundos de programas de pós-graduação. Nesse contexto, a formação de mestres e doutores bem como outras relações formais de orientação no ambiente institucional são objeto de estudo da genealogia acadêmica (Sugimoto, 2014).

Analisar a genealogia acadêmica de pesquisadores, instituições de ensino ou grupos de pesquisa possibilita analisar o papel que esses atores tiveram na construção e consolidação de comunidades científicas. Assim, é possível evidenciar relações entre as características de acadêmicos e suas produções em termos de recursos humanos (Malmgren; Ottino; Amaral, 2010; Chariker et al., 2017). Trata-se de uma forma complementar de análise àquelas já existentes baseadas na produção bibliográfica.

Quando se analisa a genealogia acadêmica de um pesquisador, por exemplo, comumente recorre-se ao uso de grafos, uma abstração matemática que representa cada objeto (acadêmico) por um círculo ou vértice, e cada relação (orientação acadêmica) entre objetos por uma reta ligando ambos os círculos (West; Vilhena, 2014). Dessa forma, um orientador está ligado aos seus orientados (filhos acadêmicos) e aos seus orientadores (pais acadêmicos) por meio de retas. Algumas medidas relacionadas à estrutura de grafos podem ser calculadas para cada vértice ou para um grupo de vértices de interesse. Entre elas está, por exemplo, o grau de um vértice, que retrata quantas relações existem entre um pesquisador e todos os outros (Albert; Barabási, 2002; West; Vilhena, 2014). Através dessas estruturas podem ser exploradas as questões relacionadas com a propagação de conhecimento e interdisciplinaridade, na perspectiva da formação de recursos humanos.

Nesse sentido, este trabalho aborda a genealogia acadêmica da Escola Nacional de Saúde Pública Sergio Arouca (ENSP), Fundação Oswaldo Cruz (Fiocruz). Os dados foram extraídos da Biblioteca da ENSP e dos currículos da Plataforma Lattes. O procedimento metodológico utilizado abrange os métodos descritivo, exploratório e analítico. O objetivo do trabalho é construir e analisar a genealogia acadêmica da ENSP, de modo a identificar suas origens e a relação existente entre a área da Saúde Coletiva e as demais áreas do conhecimento. Este trabalho tem

2 o pressuposto de que a construção do conhecimento em Saúde Pública na ENSP utilizou-se de vários campos do conhecimento, resultando em um conceito ampliado sobre o entendimento do que constitui a Saúde Pública.

\section{Trabalhos correlatos}

Alguns trabalhos utilizam grafos de genealogia acadêmica, como Mathematics Genealogy Project (s.d.) e The Academic Family Tree (2017), que investigaram relações entre pesquisadores e a estrutura de rede em que estão inseridos (Malmgren; Ottino; Amaral, 2010; Chariker et al., 2017). Outros estudos utilizam os atributos dos vértices para investigar como se dá o fluxo de conhecimento ao longo do tempo. Assim, Gargiulo et al. (2016), com base no grafo de genealogia acadêmica dos matemáticos, estudaram a evolução no tempo de atributos como disciplinas e países. 
Ainda nesse contexto, Sugimoto et al. (2011) investigaram o grau de interdisciplinaridade na área de Biblioteconomia e Ciências da Informação, nos Estados Unidos, ao longo de oitenta anos (1930 a 2009), com base em dados de mais de três mil teses na área. Os autores verificaram que essa área recebeu grande influência, nos anos iniciais, de campos como Educação e Psicologia. Mais recentemente, identificou-se uma tendência em haver menos orientadores da própria área e mais de campos como Ciência da Computação, Administração e Comunicação, demonstrando um crescente grau de interdisciplinaridade.

Em geral, os trabalhos sobre genealogia acadêmica podem ser acompanhados na internet: NeuroTree (David; Hayden, 2012); e The Academic Family Tree (The Academic Family Tree, 2017). No Brasil, a Plataforma Acácia (2018) utiliza os dados da Plataforma Lattes para construir grafos de genealogia acadêmica das orientações de pós-graduação stricto sensu (Damaceno; Rossi; Mena-Chalco, 2017). Alguns exemplos de genealogias acadêmicas construídas no Brasil são a genealogia honorífica do Professor Etelvino Bechara, do Instituto de Química (IQ) da Universidade de São Paulo (USP) (Mena-Chalco, 2015) e a genealogia histórica da área de Protozoologia (Elias; Floeter-Winter; Mena-Chalco, 2016).

O estudo ora apresentado difere-se dos demais por analisar a genealogia acadêmica de uma instituição, em particular, com base na influência entre campos do saber e em métricas em grafos, específicas para contextos de orientação acadêmica. Nas seções seguintes são apresentados os procedimentos metodológicos, os resultados obtidos, e a discussão acerca das análises realizadas. Por fim, são apresentados possíveis desdobramentos futuros deste trabalho.

\section{Procedimentos Metodológicos}

\section{Fonte de dados}

Os acadêmicos relacionados à ENSP foram identificados por meio de análise dos registros do Acervo ENSP, da Biblioteca de Saúde Pública Fiocruz. O Acervo proporciona uma série de filtros, tais como titulação (e.g., doutor ou mestre) e tipo de literatura (e.g., tese ou dissertação), entre outros. Neste trabalho, a busca selecionou tipo de literatura e, posteriormente, autores com título acadêmico igual a catedrático, doutor, livre docência, professor catedrático e professor titular. A busca retornou uma série de nomes de pesquisadores, dentre os quais parte apresentava-se com grafias diferentes, razão pela qual a listagem também passou por uma verificação manual. Os nomes dos pesquisadores orientadores relacionados aos registros do Acervo, resultantes da busca e da filtragem anteriores, foram identificados na Plataforma Lattes, para obter os respectivos Códigos Identificadores (ID) dos currículos.

\section{Construção do grafo de genealogia acadêmica}

Para construir o grafo de genealogia acadêmica da ENSP foi utilizado, com adaptações, o método desenvolvido por Damaceno, Rossi e Mena-Chalco (2017), baseado na Plataforma Lattes, que identifica o grafo de genealogia acadêmica correspondente. O método recebe como entrada os ID dos currículos dos pesquisadores e retorna como saída um grafo de genealogia composto por todos os ascendentes (advindos do campo "Formação Acadêmica") e descendentes (advindos do campo "Orientações Concluídas") de cada pesquisador. O grafo resultante desse método mostra dados de pesquisadores orientadores e seus respectivos alunos registrados no Acervo, bem como de outros pesquisadores que podem ser ascendentes ou descendentes daqueles pesquisadores e alunos. Neste trabalho foram consideradas somente as orientações de doutorado concluídas. Sobre cada pesquisador, representado por um vértice, são apresentadas informações de área e grande área de atuação, endereço profissional, formações em pós-doutorado e existência de livre-docência. Cada relação, representada por uma aresta, cuja origem é um 
orientador e destino um orientado, apresenta informações sobre anos de início e conclusão da orientação, local de recebimento do título e título acadêmico.

O grafo obtido inicialmente continha um número elevado de pesquisadores que não estavam relacionados à ENSP, dado que esse método coleta todos os ascendentes e descendentes do conjunto de pesquisadores. Dessa forma, o método foi adaptado para excluir pesquisadores e relações que não fazem parte do contexto da Instituição. O procedimento consistiu em duas etapas, sendo a primeira a classificação de cada pesquisador como semente, ascendente e/ou descendente e, posteriormente, a filtragem do grafo de pesquisadores e relações conforme essas classificações.

Cada pesquisador foi classificado como semente se estivesse na lista inicial dos currículos. Os ascendentes desses currículos foram marcados como ascendentes. Os ascendentes dos ascendentes também foram marcados dessa forma, e assim por diante. O mesmo critério foi empregado para os pesquisadores descendentes. Note-se que um mesmo acadêmico pode estar classificado como ascendente, descendente e semente, ao mesmo tempo. Foram excluídos do grafo pesquisadores representados por ascendentes de descendentes ou descendentes de ascendentes que não tivessem ligação com os pesquisadores sementes. Um resumo do processo descrito nos parágrafos anteriores é apresentado na Figura 1.

As métricas fecundidade, descendência e índice genealógico foram calculadas para todos os pesquisadores do grafo resultante. A métrica fecundidade indica o total de pesquisadores descendentes diretos (ou número de alunos orientados), a produtividade e a importância do orientador na estruturação do grafo. Descendência é a métrica em que, dado um pesquisador qualquer, é contabilizado o número de descendentes diretos e indiretos desse pesquisador, i.e., são contabilizados os filhos acadêmicos, os netos, bisnetos, trinetos e assim por diante. Finalmente, o índice genealógico (Rossi; Freire; Mena-Chalco, 2017) constitui o maior número de conexões entre um pesquisador e seus descendentes diretos que possuem, pelo menos, igual número de conexões, cada um.
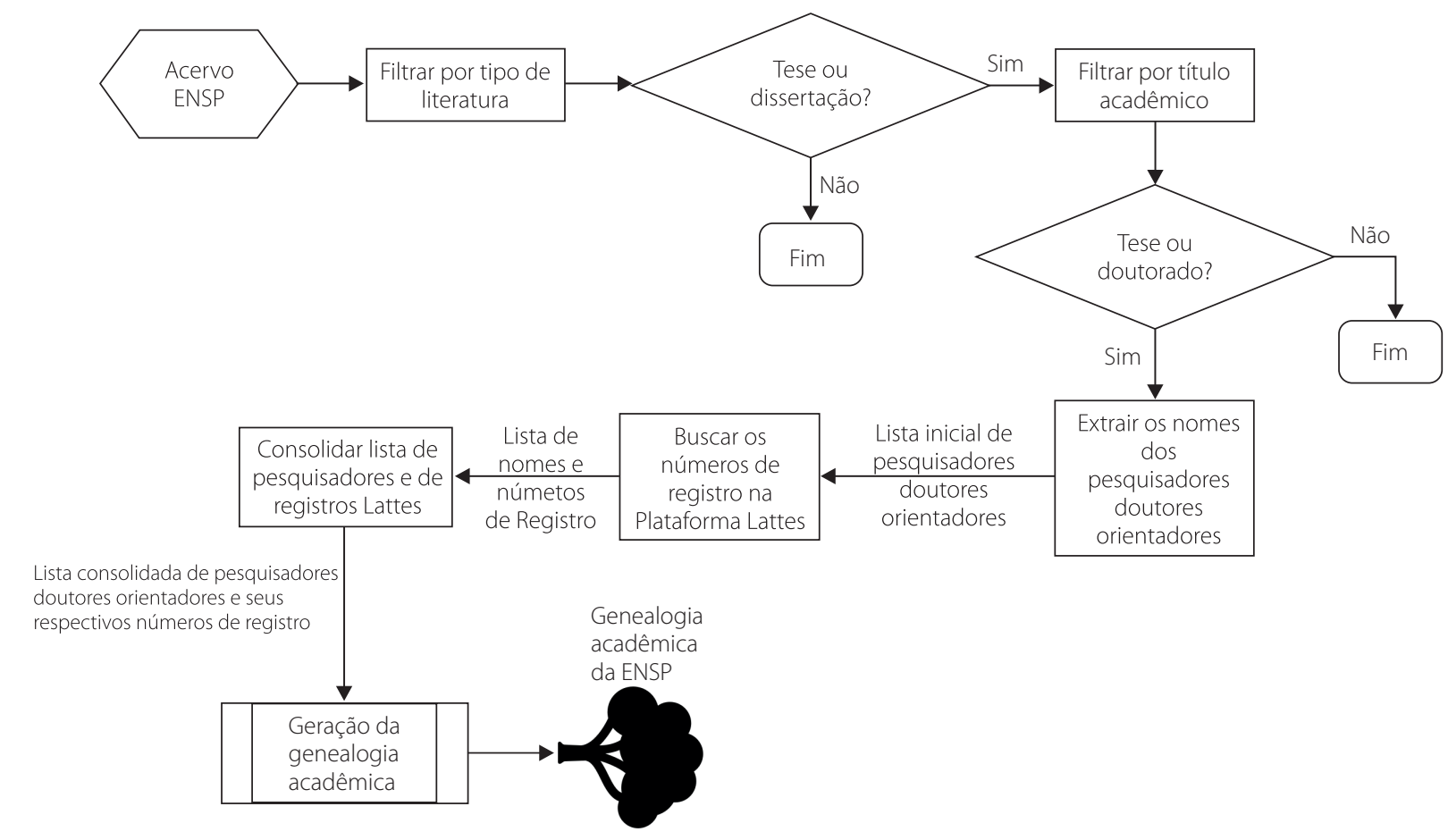

Lista consolidada de pesquisadores doutores orientadores e seus respectivos números de registro

Figura 1. Processo para geração da genealogia acadêmica dos pesquisadores doutores da Escola Nacional de Saúde Pública Sergio Arouca. Brasil, 2018.

Fonte: Elaborado pelos autores (2018). 


\section{Resultados}

A busca, realizada em dezembro de 2017 na Biblioteca da ENSP, retornou dados de 5.792 teses e dissertações. Após aplicação do filtro para titulação acadêmica igual a doutorado e afins, restaram 2.618 registros, para os quais foram identificados os respectivos ID da Plataforma Lattes. Esses registros foram consolidados, resultando em 1.464 nomes únicos de pesquisadores orientadores de doutorado, chamados de sementes neste trabalho, e que foram utilizados para construir a genealogia acadêmica da ENSP. Os pesquisadores sementes orientaram teses de doutorado na ENSP ou registraram teses de doutorado na Instituição.

O grafo mostrado na Figura 2 contém 20.959 pesquisadores e 20.516 relações de orientação. A maior parte dos pesquisadores (78\%) corresponde a acadêmicos com currículos na Plataforma Lattes, o que significa que podem ter atributos como área de atuação, endereço profissional, formações de pós-doutorado, entre outros. Cerca de 22\% dos vértices não tinham currículos nessa Plataforma e, nesses casos, os nomes podem ter sido grafados de forma diferente daquela indicada nos respectivos currículos Lattes, ou são inexistentes por se tratar de pesquisador estrangeiro ou não registrado na Plataforma Lattes.

Embora os pesquisadores da genealogia acadêmica da ENSP se distribuam por muitas instituições, 49,29\% deles estão concentrados em 25 instituições, o que corresponde a 1,15\% das mesmas, desconsiderados os vértices sem indicação de instituição. A Fiocruz e as universidades públicas se destacam como as instituições com os maiores números de pesquisadores.

Entre os pesquisadores da genealogia acadêmica da ENSP, poucos são livres-docentes (3,37\%) e somente 16,92\% fizeram pós-doutorado. Os pesquisadores sementes são o grupo com maior número relativo de livres-docentes $(16,26 \%)$ e de pós-doutores (41,46\%). A maior parte dos livres-docentes $(74,00 \%)$ trabalhava ou trabalha em universidades públicas do Estado de São Paulo. Esses números desconsideraram 8,20\% dos pesquisadores, que não indicaram vínculo institucional. A Fiocruz tem 12,00\% dos pesquisadores com estágio pós-doutoral, seguindo-se nove universidades públicas federais e estaduais.

A grande área do conhecimento das Ciências da Saúde, como esperado para a ENSP, concentra o maior número de pesquisadores, seguindo-se as áreas de Ciências Humanas, de Ciências Biológicas e de Ciências Sociais. As áreas de Ciências Exatas e da Terra, Engenharia, Ciências Agrárias e Linguística também abrigam pesquisadores da genealogia acadêmica da ENSP, porém em proporções menores que as listadas anteriormente. As áreas de Saúde Coletiva e Medicina somam perto de um quarto do total dos pesquisadores, que equivalem a quase cinco mil pessoas. Em seguida, vêm as áreas de Enfermagem, Educação e Psicologia. Completam o elenco, com participações decrescentes, mais 79 áreas do conhecimento.

A interação entre a grande área das Ciências da Saúde e as outras grandes áreas do conhecimento é apresentada na Figura 3A, o que possibilita visualizar a multidisciplinaridade na orientação acadêmica. A grande área das Ciências da Saúde é a que mais recebe influência e influencia as grandes áreas das Ciências Humanas, Ciências Biológicas e Ciências Sociais Aplicadas, i.e., os pesquisadores que atuam nessas grandes áreas foram orientados por pesquisadores que atuam em Ciências da Saúde e vice-versa.

Uma análise semelhante foi realizada no contexto das áreas do conhecimento, para verificar a influência entre Saúde Coletiva e as outras áreas. Nesse sentido, a Figura 3B mostra que a Medicina é a que mais influencia a Saúde Coletiva. Seguem-se, em termos de áreas que influenciam a Saúde Coletiva, a Sociologia, a Imunologia, a Ciência e Tecnologia de Alimentos, a Parasitologia, a Antropologia, a Farmacologia e a História. Na outra direção, as áreas de Enfermagem, Nutrição e Administração são as mais influenciadas pela Saúde Coletiva, que mantém relação equilibrada com a Odontologia e a Psicologia. 


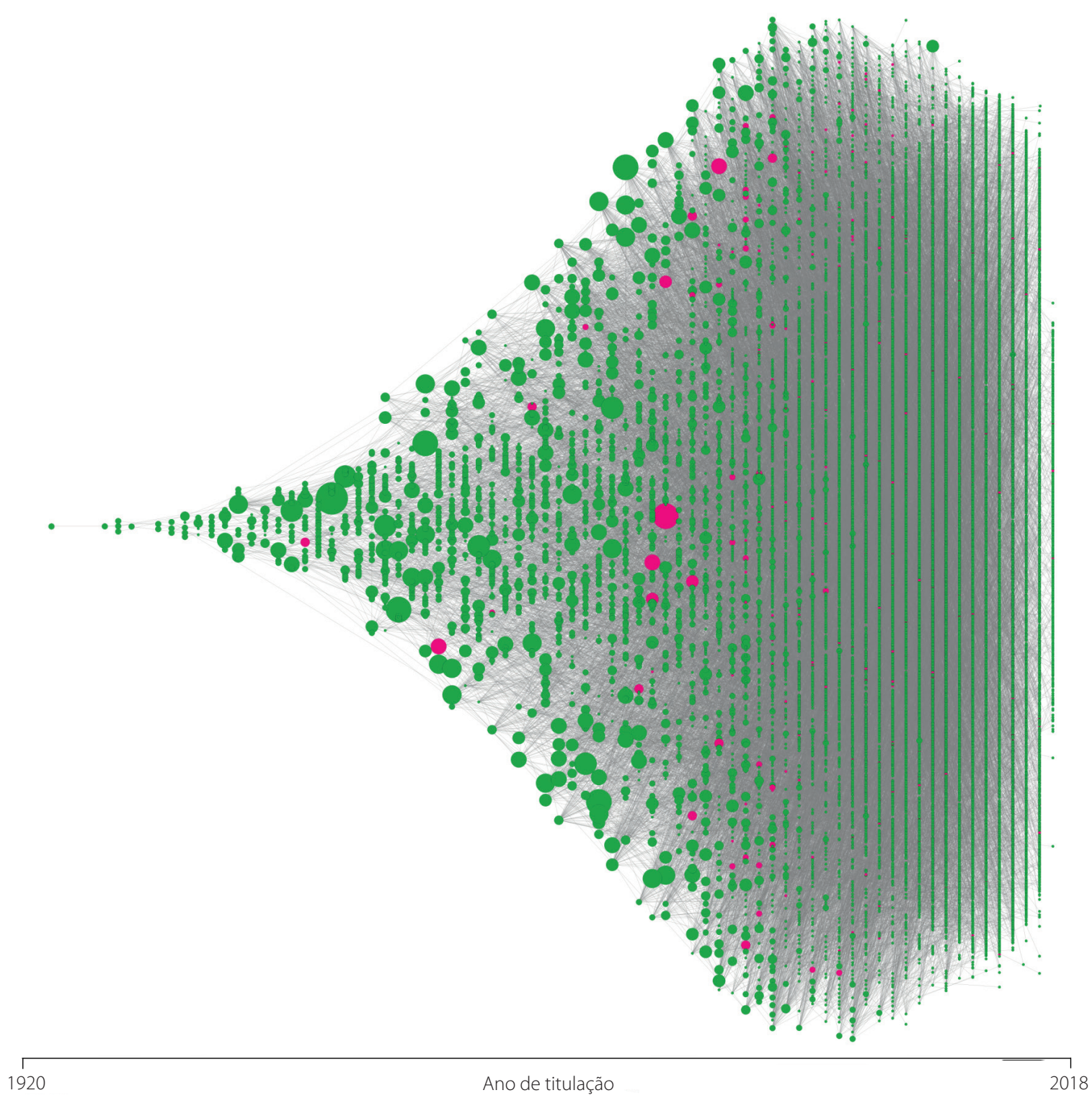

Figura 2. Genealogia acadêmica dos pesquisadores doutores da Escola Nacional de Saúde Pública Sergio Arouca. Brasil, 2018.

Fonte: Elaborado pelos autores (2018).

Para além das grandes áreas eáreas do conhecimento, as métricas calculadas para o conjunto de pesquisadores indicaram fecundidade média igual a 1 e máxima igual a 68; valores médio e máximo para descendência, 3 e 518; e, para índice genealógico, média igual a 0,12 e máxima 9. Nota-se que as diferenças entre a média e o máximo para todas as métricas é acentuada, em especial para a descendência.

A descendência representa a produtividade e a importância do pesquisador na estruturação de um grafo. Dois pesquisadores que fizeram suas carreiras na ENSP estão presentes entre aqueles com maior número de descendentes diretos e indiretos: Vincent Valla, com 319 descendentes, e Maria Cecilia de Souza Minayo (orientada por Vincent Valla), com 265. Ambos os pesquisadores atuaram e atuam na Saúde Coletiva. A pesquisadora Maria Cecilia de Souza Minayo, da ENSP, também está entre os 25 pesquisadores mais relevantes do ponto de vista do número de orientações diretas de doutorado em Saúde Coletiva, que corresponde à métrica de fecundidade. 
Influência entre Ciências da Saúde e outras Grandes Áreas

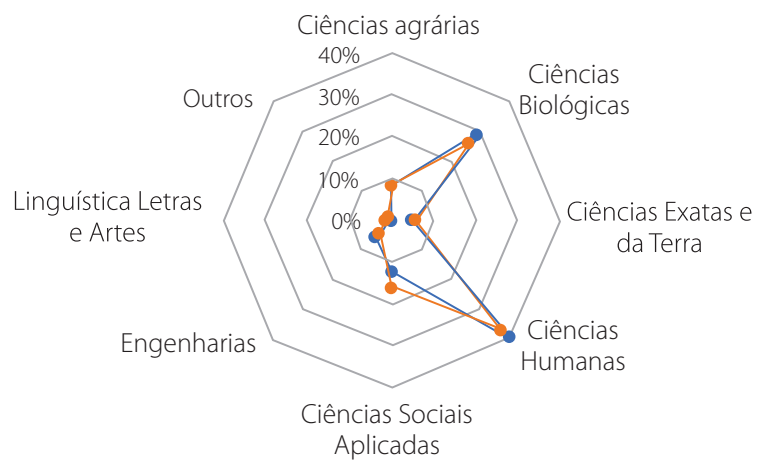

- Orientações realizadas $\longrightarrow$ - Orientações recebidas

(a) Grandes Áreas do conhecimento
Influência entre Saúde Coletiva e outras Áreas

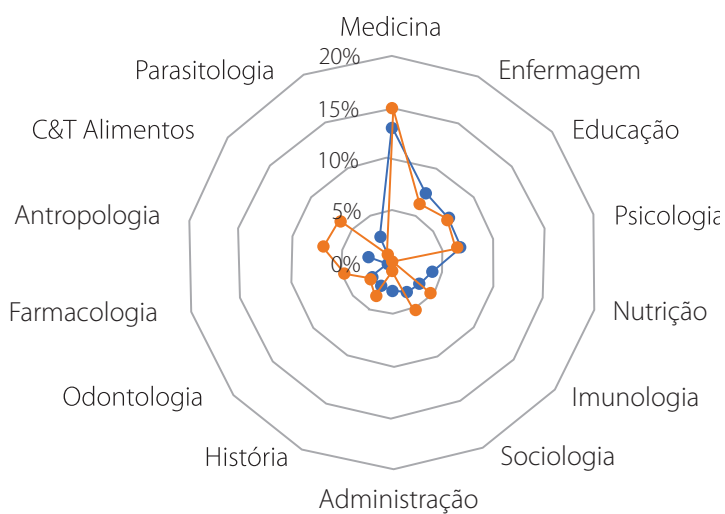

- Orientações realizadas —O Orientações recebidas

(b) Áreas do conhecimento

Figura 3. Influência entre Ciências das Saúde e outras Grandes Áreas (a) e entre Saúde Coletiva e outras Áreas (b). Brasil, 2018.

Fonte: Elaborado pelos autores baseado nas informações de genealogia acadêmica da Escola Nacional de Saúde Pública Sergio Arouca (2018).

Os valores do índice genealógico fornecem uma avaliação quali-quantitativa da genealogia acadêmica da ENSP. Como destaque, na Figura 4, apresentam-se dois pesquisadores muito influentes na orientação de acadêmicos: a professora Maria Cecilia Minayo, que possui o maior índice genealógico (orientou sete alunos de Doutorado que, por sua vez, orientaram, pelo menos, sete alunos de doutorado, cada um), e o professor Luiz Fernando Rocha Ferreira da Silva, que é o mais antigo pesquisador da ENSP, ou seja, o primeiro acadêmico a formalmente se transferir para a ENSP, na década de 1960.

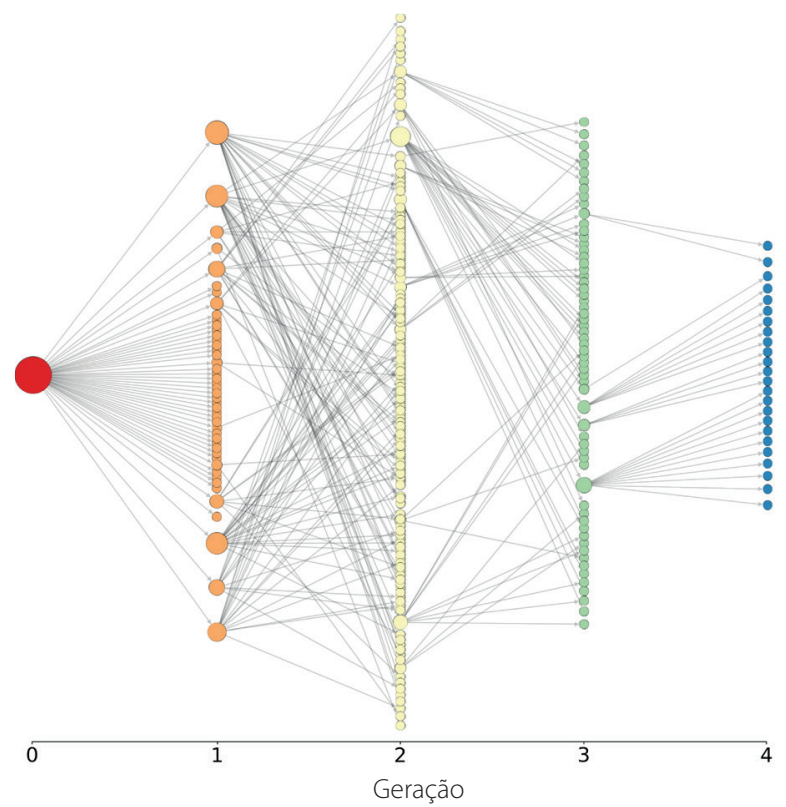

(a) Maria Cecilia de Souza Minayo
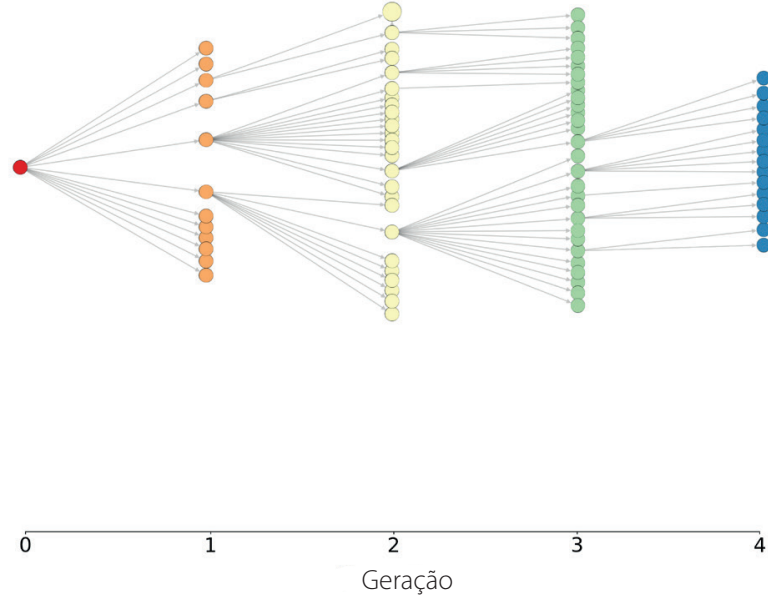

(b) Luiz Fernando Rocha Ferreira da Silva

Figura 4. Representação em grafo da genealogia acadêmica da professora Maria Cecilia Minayo e do professor Luiz Fernando Rocha Ferreira da Silva. Brasil, 2018.

Fonte: Elaborado pelos autores, com base nas informações de genealogia acadêmica da Escola Nacional de Saúde Pública Sergio Arouca (2018). 


\section{Discussão}

Um primeiro olhar sobre os resultados aqui apresentados já confirma que o trabalho permite aportar informações novas à interpretação da trajetória desenvolvida pela ENSP ao longo de suas mais de seis décadas de existência. Apesar de a abordagem metodológica não ter pretendido levar em conta a história, o crescimento e os processos de transformação institucional, observa-se que os resultados podem ser relacionados ao que empiricamente é conhecido, ou ao que já vem sendo apontado por alguns autores que se debruçaram sobre a trajetória da Instituição (Lima; Fonseca; Santos, 2004).

Os pesquisadores da genealogia acadêmica da ENSP trabalham, majoritariamente, em institutos públicos de pesquisa e universidades públicas, estaduais e federais, como também fizeram seus cursos de Doutorado nessas mesmas instituições. Nesse sentido, a participação do setor privado na formação e na absorção de profissionais qualificados para a área da Saúde Coletiva mostrou-se reduzida e de pouca expressão na composição da genealogia acadêmica da ENSP.

A Fiocruz é a instituição com maior contingente de pesquisadores (orientadores e orientados), inclusive entre os que realizaram pós-doutorado. Destaque-se, ainda, a presença de numerosas instituições que abrigam os pesquisadores da genealogia acadêmica da ENSP, indicando a existência de cooperação institucional e de troca de conhecimentos. Entretanto, como já visto, há uma concentração de pesquisadores em um número reduzido de instituições, localizadas nas Regiões Sudeste e Sul do País. Tal fato também foi observado por Sidone, Haddad e Mena-Chalco (2016), que estudaram a distribuição geográfica de publicações e colaborações, considerando a filiação institucional dos pesquisadores. Para os autores do estudo, tal distribuição resulta, em parte, da concentração de institutos de pesquisas públicos e de universidades públicas nessas Regiões e em suas capitais estaduais, as quais têm maior capacidade de captação de recursos financeiros e de pessoal qualificado.

A proporção entre o número de pesquisadores e as relações de orientação de doutorado - 20.959 para 20.516 - é semelhante à proporção da genealogia acadêmica dos doutores registrados na Plataforma Lattes (Damaceno; Rossi; Mena-Chalco, 2017), igual a 372.284 pesquisadores e 387.246 relações de orientações. Entretanto, contrasta com a proporção entre os doutores atuantes em Matemática no Brasil (4.906) e as relações de orientação de doutorado (8.025), conforme apontam Rossi et al., 2018.

O conjunto da genealogia acadêmica da ENSP apresentou resultado equilibrado entre orientações recebidas e realizadas. Esse resultado também foi observado nas Ciências da Saúde, que são quase igualmente influentes e influenciadas pelas demais grandes áreas, sem ocorrer diferença expressiva entre orientações realizadas (6.288) e recebidas (6.054). Os dados mostraram que as Ciências da Saúde são ligeiramente mais influentes nas Ciências Biológicas, nas Ciências Humanas, nas Engenharias e nas Ciências Agrárias. O papel influenciador das Ciências da Saúde também foi verificado por Rossi et al. (2018). De outro modo, as Ciências Sociais Aplicadas, as

8 Ciências Exatas e da Terra, bem como Linguística, Letras e Artes, pouco influenciam as Ciências da Saúde. Essa troca de conhecimentos entre grandes áreas e áreas do conhecimento pode ser considerada um marcador da multidisciplinaridade em Saúde Coletiva, embora ainda não exista um consenso sobre os limites de multi, inter e transdisciplinaridade (Bicalho, 2009; Maricato; Reis, 2017).

Embora o conjunto da Saúde Coletiva oriente mais e, portanto, influencie mais as demais áreas do conhecimento, há diferenças entre estas e aquela. Assim, o número de orientações realizadas e recebidas é equilibrado entre a Saúde Coletiva e as áreas de Educação, Odontologia e Psicologia. Além da Medicina, as áreas de Enfermagem, Educação e Nutrição são aquelas que mais influenciam a Saúde Coletiva.

É importante ressaltar que a influência de campos do conhecimento sobre os demais parece modulada por vários fatores, como a capacidade dos pesquisadores em produzir resultados mensuráveis, habilitando-se como 
orientadores de Programas de Pós-Graduação. Também se mostra relevante a capacidade do pesquisador em atrair e absorver alunos de Pós-Graduação, gestando novas gerações de pesquisadores.

Há muitos pesquisadores relevantes, a depender da dimensão que se queira destacar, mas dois deles sobressaem: Luiz Fernando Rocha Ferreira da Silva, pesquisador que inaugurou as Ciências Biológicas na ENSP, em 1966, e Maria Cecilia de Souza Minayo, que experimenta valores expressivos nas métricas selecionadas de descendência, fecundidade e índice genealógico, estando presente entre os 25 pesquisadores mais relevantes. A Figura 4 apresentou os grafos da genealogia acadêmica dos dois pesquisadores.

O ramo de Parasitologia, área de atuação do professor e pesquisador Luiz Fernando Rocha Ferreira da Silva, é o mais antigo da ENSP, e reporta-se ao professor de Medicina da Universidade Federal do Rio de Janeiro (UFRJ), José Rodrigues da Silva (Sociedade Brasileira de Medicina Tropical, 2014), que, no final da década de 1940, obteve a Livre Docência em Clínica Médica (Esquistossomose). Posteriormente, José Rodrigues da Silva orientou, em 1962, o doutorado do pesquisador Luiz Fernando Rocha Ferreira da Silva, na UFRJ. Antes pesquisador titular, ele é atualmente professor emérito da Fiocruz, e seu ramo de pesquisas encontra-se na sexta geração de doutores na ENSP.

Seguindo por antiguidade, em 1961 foi outorgada a Livre Docência em Medicina (Chagas) a Guilherme Rodrigues da Silva, professor da Universidade Federal da Bahia (UFBA), que em meados da década de 1960 se transferiu para a USP, retornando mais tarde para a UFBA (Goldbaum, 2015). Entre os descendentes desse professor, foram identificados dois pesquisadores sementes da ENSP que defenderam suas respectivas teses de doutorado em 1988 e 1997, nas áreas de Epidemiologia Ambiental/Epidemiologia do Câncer (Sergio Koifman) e Epidemiologia Materno-Infantil (Maria do Carmo Leal), respectivamente. Esses pesquisadores sementes da ENSP orientaram novas gerações de doutores, iniciando uma ramificação numerosa em campos do conhecimento da Epidemiologia.

Em 1964 foi concedido o título de Livre Docente, em História, a Nícia Vilela Luz, professora da USP (Capelato; Glezer; Ferlini, 1994), que tem entre seus descendentes diretos Victor Vincent Valla, e indiretos Maria Cecília de Souza Minayo, os quais, por sua vez, têm as maiores métricas de descendência da genealogia acadêmica da ENSP. As áreas do conhecimento de atividade desses pesquisadores e seus descendentes são Educação, e Violência e Saúde.

Ainda, no fim da década de 1960, ocorreu o doutoramento, na Espanha, em Química, e na USP, em Engenharia Sanitária, de dois pesquisadores sementes, que estão na origem das áreas de Saúde do Trabalhador e Ecologia Humana, e de Saneamento e Saúde Ambiental na ENSP.

\section{Considerações Finais}

As métricas utilizadas neste trabalho retratam o pesquisador típico do grafo da genealogia acadêmica da ENSP como sendo aquele que tem três descendentes diretos e indiretos, orientou apenas um aluno de doutorado, e tem índice genealógico menor que um. Esse pesquisador típico reflete a lei de potência, conforme a qual um número elevado de pesquisadores não orientou alunos de doutorado e poucos pesquisadores orientaram muitos alunos de doutorado, conforme mostram as métricas do grafo da ENSP: fecundidade máxima igual a 68, descendência maior que 500 e índice genealógico igual a 9.

A análise dos pesquisadores mais antigos do grafo identificou a influência de diferentes campos do conhecimento na constituição e na consolidação acadêmica da ENSP. Essa origem diversa contribuiu para o fortalecimento de um conceito de Saúde Pública como o resultado da interação entre condições socioeconômicas, culturais e ambientais, que vai além do biológico: envolve prevenção, nutrição, saneamento, moradia e qualidade de vida.

A realização deste trabalho demonstrou o potencial do conceito de grafos e de genealogia acadêmica para a análise da comunidade científica institucional. O procedimento metodológico empregado no presente estudo 
poderá guiar estudos genealógicos em outros campos do conhecimento. Desdobramentos deste trabalho poderão ampliar o estudo sobre a genealogia acadêmica da Saúde Coletiva no Brasil.

\section{Colaboradores}

L.C.S. Viana e J.P. Mena-Chalco participaram na elaboração de estratégia experimental. L.C.S. Viana, J.P. Mena-Chalco e R.J.P. Damaceno participaram coleta de dados, tabulação e discussão dos resultados e elaboração do artigo. S.M.F.M. Souza participou na elaboração do projeto de pesquisa, tabulação e discussão dos resultados e elaboração do artigo.

\section{Referências}

Albert, R.; Barabási, A.L. Statistical mechanics of complex networks. Reviews of Modern Physics, v.74, n.1, p.47-97, 2002

Bicalho, L.M. As relações interdisciplinares refletidas na literatura brasileira da ciência da informação. 2009. 268f. Tese (Doutorado em Ciência da Informação) - Universidade Federal de Minas Gerais, Belo Horizonte, 2009.

Capelato, M.H.R.; Glezer, R.; Ferlini, V.L.A. Escola uspiana de História. Estudos Avançados, v.8, n.22, p.349-358, 1994. Disponível em: http://www.revistas.usp.br/eav/article/view/ 9719. Acesso em: 12 set. 2017.

Chariker, J.H. et al. Identification of successful mentoring communities using network-based analysis of mentor-mentee relationships across Nobel laureates. Scientometrics, v.111, n.3, p.1733-1749, 2017.

Damaceno, R.J.P.; Rossi, L.; Mena-Chalco, J.P. Identificação do grafo de genealogia acadêmica de pesquisadores: uma abordagem baseada na Plataforma Lattes. In: Brazilian Symposium on Databases, 32., Uberlândia. Proceedings [...] Uberlândia: SBBD, 2017. Disponível em: http://sbbd.org. br/2017/wp-content/uploads/sites/3/2017/10/proceedingssbbd-2017.pdf. Acesso em: 15 set. 2018.

David, S.V.; Hayden, B.Y. Neurotree: A collaborative, graphical database of the academic genealogy of neuroscience. Plos One, v.7, n.10, e46608, 2012. Available from: http://dx.plos. org/10.1371/journal.pone.0046608. Cited: Aug. 1, 2017.

Elias, M.C.; Floeter-Winter, L.M.; Mena-Chalco, J.P. The dynamics of Brazilian protozoology over the past century. Memórias do Instituto Oswaldo Cruz, v.111, n.1, p.67-74, 2016. Available from: http://www.scielo.br/scielo.php?script=sci_ arttext\&pid=S0074-02762016000100067\&lng=en\&nrm=iso. Cited: Aug. 4, 2017.

Gargiulo, F. et al. The classical origin of mathematics. EPJ Data Science, v.5, n.1, p.1-15, 2016. Available from: https:// epjdatascience.springeropen.com/articles/10.1140/epjds/ s13688-016-0088-y. Cited: Aug. 1, 2017.

Goldbaum, M. Guilherme Rodrigues da Silva: a formação do campo da Saúde Coletiva no Brasil. Ciência e Saúde Coletiva, v.20, n.7, p.2129-234, 2015. Disponível em: http://www.scielo. br/scielo.php?script=sci_arttext\&pid=\$1413-81232015000 702129\& lng=en\&nrm=iso. Acesso em: 12 set. 2017.
Lima, N.T.; Fonseca, C.M.O.; Santos, P.R.E. Uma escola para a saúde. Rio de Janeiro: Editora Fiocruz, 2004. E-book. Disponível em: http://books.scielo.org/id/d48x7. Acesso em: 1 ago. 2018.

Malmgren, R.D.; Ottino, J.M.; Amaral, L.A.N. The role of mentorship in protégé performance. Nature, v.465, p.622-626, 2010.

Maricato, J.M.; Reis, F. Relações interdisciplinares entre a Ciência da Informação e a Ciência da Comunicação: uma análise a partir de citações, formação das bancas e palavras-chave das teses das áreas. Perspectiva em Ciência da Informação, v.22, n.1, p.112-132, 2017. Disponível em: http:// www.scielo.br/scielo.php?script=sci_arttext\&pid=\$1413-993 62017000100112\&lng=en\&nrm=iso. Acesso em: 1 ago. 2017.

Mathematics genealogy project. Dakota: North Dakota State University, [s.d]. Available from: https://www.genealogy.math. ndsu.nodak.edu/index.php. Cited: Sep 3, 2018.

Mena-Chalco, J.P. A genealogia acadêmica do Prof. Etelvino José Henriques Bechara: relatório de divulgação científica. São Paulo: Universidade Federal do ABC, 2015. Disponível em: https://search.datacite.org/works/10.13140/RG.2.1.1095.3685. Acesso em: 2 ago. 2017.

Plataforma Acácia. Genealogia acadêmica do Brasil. Universidade Federal do ABC, 2018. Disponível em: http://plataformaacacia.org/. Acesso em: 9 set. 2018.

Rossi, L. et al. Genealogia acadêmica dos doutores atuantes em matemática: um mapeamento macro na ciência brasileira. Proceeding Series of the Brazilian Society of Computational and Applied Mathematics, v.6, n.1, 2018. Disponível em: https:// proceedings.sbmac.org.br/sbmac/article/view/2182. Acesso em: 15 set. 2018.

Rossi, L.; Freire, I.L.; Mena-Chalco, J.P. Genealogical index: A metric to analyze advisor-advisee relationships. Journal of Informetrics, v.11, n.2, 2017, p.564-582. Available from: https://www.sciencedirect.com/science/article/pii/S175115 7716302632. Cited: Aug. 1, 2017.

Sidone, O.J.G.; Haddad, E.A.; Mena-Chalco, J.P. A ciência nas regiões brasileiras: evolução da produção e das redes de colaboraçãocientífica. Transinformação,v.28, n.1, p.15-32,2016. Doi: http://dx.doi.org/10.1590/2318-08892016002800002. Disponível em: http://www.scielo.br/scielo.php?script=sci_ arttext\&pid=S0103-37862016000100015\&lng=en\&nrm=iso. Acesso em: 1 ago. 2017. 
Sociedade Brasileira de Medicina Tropical. Ex-presidentes. Brasília: SBMT, 2014. Disponível em: http://www.sbmt.org.br/ portal/presidentes/. Acesso em: 12 set. 2017.

Sugimoto, C.R. Academic genealogy. In: Croning, B.; Sugimoto, R. (Ed.). Beyond bibliometrics: Harnessing multidimensional indicators of scholarly impact. Cambridge: The MIT Press, 2014. p.365-382.

Sugimoto, C.R. et al. Academic genealogy as an indicator of interdisciplinarity: An examination of dissertation networks in Library and Information Science. Journal of the American
Society for Information Science and Technology, v.62, n.9, p.180818-180828, 2011.

The Academic Family Tree: Building a single, interdisciplinary academic genealogy. 2017. Available from: https:// academictree.org/. Cited: Sept. 3, 2018.

West, J.D.; Vilhena, A.V. A network approach to scholary evaluation. In: Cronin, B.; Sugimoto, C.R. (Ed.). Beyond bibliometrics: Harnessing multidimensional indicators of scholarly impact. Cambridge: MIT Press, 2014. p.51-65. 